\title{
The Meaning of Obesity among Marriage and Family Therapists
}

Moore $\mathbf{D}^{1^{*}}$, Williams $\mathrm{L}^{2}$, Kimbrough $\mathbf{A}^{3}$, Taylor $\mathbf{C}^{4}$, Jacob $\mathbf{M}^{4}$ and Willis $\mathbf{M}^{5}$

${ }^{1}$ Alliant International University, San Francisco Campus, USA

${ }^{2}$ College of Pharmacy, Xavier University of Louisiana, USA

${ }^{3}$ Georgia Military College, Columbus, Georgia, USA

${ }^{4}$ Mercer University School of Medicine, USA

${ }^{5}$ Department of Marital \& Family Therapy, California State University, Dominguez Hills, USA

"Corresponding author: Moore DD, Associate Professor and Site Program Director, Alliant International University, San Francisco Campus, USA, Tel: 6122963758; Email: darren.moore@alliant.edu

Received date: September 28, 2016; Accepted date: November 2, 2017; Published date: November 6, 2017

Copyright: (C) 2017 Moore D, et al. This is an open-access article distributed under the terms of the Creative Commons Attribution License, which permits unrestricted use, distribution, and reproduction in any medium, provided the original author and source are credited.

\begin{abstract}
In this study, researchers examined the meaning of obesity among marriage and family Therapists utilizing a qualitative methodological approach (phenomenology). A total of 15 MFTs engaged in semi-structured interviews and reflected on their epistemology regarding obesity and weight-related concerns among individuals, couples and families, in addition to reflecting on their level of preparation regarding working with obese clients in clinical treatment. This study illuminated three themes: 1. Being unhealthy, being overweight; 2 . personal knowledge and self-perceptions; and 3. Lack of knowledge and confidence. In this study, authors discuss clinical implications as well as provide recommendations regarding obesity training for therapists.
\end{abstract}

Keywords: Obesity; Marriage and family therapy; Clinical intervention

\section{Introduction}

Obesity is an issue that impacts individuals, couples, and families, here in America and abroad, and has been recognized as a national epidemic since 1999 when the centers for disease control and prevention (CDC) published a series of maps that exposed the issue's prevalence [1]. In the following years, many health organizations started to categorize obesity as a disease and the American medical association officially recognized it as such in 2013 [2,3]. The most recent CDC obesity data brief prepared by Ogden et al. reported that just over $36 \%$ of adults and $17 \%$ of youth in the United States fall into the obese category [4].

Despite the extensive discussion of obesity within scholarly literature, a significant amount of the literature has focused on health professionals and medical interventions used to induce weight loss and relinquish associated comorbidities [obstructive sleep Apnea (OSA), Type 2 diabetes mellitus (T2DM), hypertension, hypercholesterolemia, various cancers, and heart disease, among others] [5]. However, paucity exists in the discussion regarding the role and training of behavioral specialists, such as marriage and family therapists (MFTs) who may work with obese populations. Arguably, marriage and family therapists may be uniquely qualified to assist clients who struggle with the psychological, psychosocial, and relational aspects of obesity, weight loss, eating disorders, body image, and other weight-related concerns, given their attention to systemic, relational, and biopsychosocial perspectives towards clinical treatment [6].

While a few Medical Family Therapy (MedFT) training programs have emerged (master's programs, post-master's certificate programs, and doctoral programs) as well as a couple of MFT programs that are uniquely housed in a medical school or nursing school, the extent to how meticulous these programs are in training specifically on the topic of obesity is unknown. Likewise, though some traditional programs may offer a health-related elective, obesity is not a topic that is covered thoroughly in traditional MFT programs (non-MedFT) based on a review of core courses in COAMFTE-accredited master's programs, which serves as the primary mechanism for training MFTs in the united states. Consequently, these limits the exposure graduates have to proper training and methodologies in addressing the needs of this population. Obese clients often experience medical, psychological, social, and emotional impacts that instructors do not readily discuss in courses or trainings aimed at addressing diversity within couple and family relational systems. Additionally, how graduates perceive obesity, which can ultimately influence their work, does not receive the necessary attention.

While some students may gain exposure and training specifically in working with obese populations during their internship experiences, the standard preparation process for potentially working with this population during the didactic portion of these students' clinical training remains unclear. With the exception of a few studies [6-8], there has been a lack of discussion regarding the training methods used to prepare marriage and family therapists to work with obese populations. In addition, the existing work has been focused on perspectives of treatment, with limited exploration regarding epistemology, knowledge, and perceptions of obesity as a phenomenon and as a clinical treatment issue. The researchers utilized this study to explore how MFTs, as individuals and as members of the MFT field, construct meaning around the topic of obesity. Essentially, this study is about epistemology: What one knows (about obesity) and how that individual knows what she/he knows. While previous researchers have assessed how marriage and family therapists think about treatment utilizing quantitative measures, to date there has not been a study that specifically explores what marriage and family therapists know about obesity in terms of their general knowledge. While it is known that MFTs have a unique lens to treat obesity and conceptualize how 
obesity fits within family systems [6], what is not known is information about marriage and family therapists' epistemology as it relates to obesity. Arguably, epistemology informs therapists' attitudes, perceptions, and beliefs, which all may have implications for clinical treatment. Therefore, exploring this topic may inform the future of MFT training. The research may illuminate gaps or potential shortcomings in general knowledge of obesity that may merit attention in educational programs.

\section{Existing Literature}

\section{Obesity treatment and research in marriage and family therapy}

Researchers in the mental health field have found that in addition to physical health concerns, obesity also impacts mental health and quality of life in general. For example, research has shown a correlation between obesity and depression [9], though further research is needed to determine the exact direction of the relationship. In addition, researchers have noted that obesity contributes to negative lifestyle outcomes (self-stigma, shame, and perceptions of enduring discrimination, along with an overall diminished quality of life) $[10,11]$.

The field of Marriage and Family Therapy has emphasized the role of the family in regards to the issue of obesity. According to Gibson et al. [12], family members of individuals with obesity play a vital role in supporting weight-loss efforts and behavior change. Some investigators support talk therapy and see this as a crucial step in order for professionals to have an understanding of family structure and dynamics, behavioral functioning, emotional coping skills, comorbidity, and motivation when working with an obese client [13]. Researchers in the field of marriage and family therapy as well as human development, family studies, and medicine have noted the effectiveness of marriage and family therapy in working with clients regarding the matter of obesity [14-17]. Furthermore, a significant number of researchers have found that family-based interventions are effective for the treatment of obesity [18]. However, family-based interventions may or may not be conducted by marriage and family therapists [19].

While some scholars have discussed obesity within the MFT field $[20,21]$ obesity among children, obesity and bariatric surgery patients [22-26], along with other obesity research conducted by MFTs [27], a lack of discussion exists regarding how MFTs specifically define and understand obesity from a personal and clinical perspective. Furthermore, the discussion of obesity within the field of MFT has not been substantive beyond individuals reporting that they apply a "systemic lens" to the issue of obesity. In addition, the field still lacks a utilitarian definition and understanding of obesity.

In a study by Pratt et al. a majority of Marriage and Family Therapists reported the use of cognitive behavioral therapy and solution-focused therapy to conceptualize working with obese populations. However, how therapists specifically apply these theories remains unknown. Other theories or frameworks discussed in the field of MFT related to obesity, such as systemic, FIRO approach, structural, bowenian, and strategic, among others emerged in research published over 15 to 20 years ago [28-32] coupled with a limited discussion regarding application in recent years. Also, a dearth of discussion exists regarding how MFT educators utilize, teach, and assess for knowledge regarding some of the above-mentioned theories. Furthermore, self-of- the therapist or personal knowledge and experiences with obesity have not been discussed in the literature utilizing qualitative methodologybeyond open-ended questions on a survey.

\section{Obesity education in MFT}

In a review of accredited Master's programs, authors found only a few programs-master's, post-Master, and doctoral-that offered certificates and degrees in medical family therapy that explicitly addressed the issue of obesity. However, in a review of the curriculum of both traditional and MedFT programs, the focus remains on processes of therapy and collaboration, rather than on discussions and training regarding specific medical illnesses such as obesity, oncology, and infertility, among others [33].

Marriage and Family Therapists work with obese populations and increasingly work in collaborative healthcare settings [34]. Despite this fact, little information exists regarding the training procedures for master's students, in relation to specific medical illnesses. In a study conducted by Pratt et al. of MFTs regarding weight-related behaviors among MFT students and therapists, researchers found that the majority of therapists lacked training in working with obese individuals. In this study, therapists also remained unclear as to how to relate family systems theory to the clinical treatment of patients dealing with weight loss and other weight-related behaviors. Likewise, other researchers have indicated that weight bias education may be needed within marriage and family therapy training programs as an increasing number of therapists interact with obese populations. For example, Pratt et al. [7] conducted a study among 162 marriage and family therapy students and found that some therapists present an explicit weight bias. If marriage and family therapy educators do not adequately train students on how to broach working with obese clients, they may adversely impact client outcomes (engagement in treatment and adherence to treatment among therapists and other medical professionals) when they matriculate into the field. In addition, other researchers have explored the topic of weight bias in the field of marriage and family therapy and have suggested that it definitely deserves attention in the process of training therapists to work in the field of obesity and weight-related behaviors [7]. The focus of this study was to explore the meaning of obesity among Marriage and Family Therapists-specifically to explore epistemology as it relates to obesity among Marriage and Family Therapists. The researchers focused on exploring what marriage and family therapists know about obesity and how they know what they know.

\section{Methodology}

The authors employed qualitative research methods as the main purpose of the study in order to gain in-depth information regarding the topic of obesity among MFTs. In particular, the authors incorporated phenomenology in efforts to capture how MFTs construct meaning regarding obesity and to understand MFTs' experiences in the course of their graduate training programs. The MFT field has utilized phenomenology as an interpretive inquiry and has incorporated this approach as a mechanism to understand the construction of meaning [35]. This study's researchers utilized phenomenology to assess how marriage and family therapists construct meaning. The researchers formulated the following overarching research question for the study: "what is the meaning of obesity among marriage and family therapists?" subsequent questions for the study included: 1 . "What do marriage and family therapists know about obesity?" and 2. "What have MFTs learned about obesity 
during their graduate education?" This study's researchers integrated these questions and this methodological approach to examine both current knowledge and experiences transpiring within the graduate level training of future MFTs.

\section{Research Design}

This study aims to explore marriage and family therapist's perspectives regarding the topic of obesity. The authors attempted to gain an in-depth understanding of the subject matter and, therefore, elected to conduct individual interviews with participants. In the study, individuals participated in a 45 to 60 minute semi-structured interview conducted by the Primary Investigator or other co-investigators. The authors used a semi-structured interview format to allow for flexibility and an organic interview [36]. Semi-structured interviews afforded the researchers the opportunity to ask probing questions as well as followup questions that may not have been allowed using a standardized interview protocol. The use of probing questions, prompts, and followup questions resulted in an in-depth exploration of MFTs' experiences and perspectives congruent with the utilization of phenomenological approaches [37].

Due to the nature of the study and efforts to gain access to MFTs from across the nation, the researchers conducted phone interviews. While other technology such as Skype has been used in previous studies [38], the authors wanted to ensure that not having access to computer technology in respondent's private homes would not pose a barrier to participating in research. Phenomenologists note the importance of exploring topics in their natural environment and stress the significance of respondents being comfortable when participating in research [34]. Therefore, the authors employed the use of telephone interviews to maximize the comfort of the study's participants since they can use telephones at home, in the workplace, or any other setting. Likewise, telephone interviews do not require connection to the Internet, which some participants may not have in their home environment. The authors selected individual interviews to ensure that respondents have the freedom and flexibility of answering questions honestly without due influence of others in a focus group. In particular, the authors wanted to omit any opportunities for the Hawthorne Effect, social desirability, or the "group effect" present within the context of larger interviews or focus groups [39]. Qualitative research determines sample size based on the concept of saturation. Saturation occurs when no new themes emerge in the data [40]. In this study, interviews continued until the questions resulted in no new information.

\section{Credibility and Transferability}

Two important aspects of qualitative research design include credibility and transferability. Credibility refers to the trustworthiness of the research, specifically relating to both the methods and results [41]. The researchers incorporated credibility through the use of member-checks; after conducting the interviews, researchers noted any questions that emerged after finishing the data collection. To ensure credibility, the researchers relied on the utilization of multiple coders (consensus). The research team also utilized reflexivity during the research process, a tool that that identifies the positionality of researchers as well as potential biases that may exist [42]. Before starting the research study, members composed a narrative about their perspectives and epistemology regarding the topic of obesity, and then discussed this narrative with the PI. The research team members also notated their experiences and reactions while immersed in the research study to ensure that they monitored their own perspectives in relation to the research subject. A brief review of each team member's credentials follows:

The first author is an associate professor and site director of an MFT program, as well as a licensed marriage and family therapist. His area of research is obesity and weight loss, related to individuals, couples, and families, with an emphasis on health disparities among men, African American families, and other unique or marginalized populations. He has developed courses regarding the topic of obesity within an undergraduate program and has integrated obesity education within some of his work teaching in an MFT and medical school program. The second researcher is an assistant clinical professor of pharmacy in a graduate program at a university in the southern United States. Her research focus includes health disparities and pharmacy education, as well as inter-professional education and collaboration. The third researcher is an assistant professor at an undergraduate institution as well as a multi-systemic therapist. Her research interest's span clinical supervision, health and mental health, and working with youth and families, as well as working with child and adult criminal offenders. The fourth researcher is an assistant professor in a marriage and family therapy graduate program on the west coast. Her research interests include health disparities with an emphasis on autism spectrum disorders, health, and minority student education. The fifth author is an advanced marriage and family therapy student at an institution in the south, and she has an interest and research focus on health disparities among women in marginalized populations. The sixth author is a third year medical student at a school in the South, and his research focuses on the relationship between bariatric surgery patients and marriage and family therapists. In addition to reflexivity assignments, the researchers employed the use of bracketing throughout the research process [43].

Another important aspect of research hinges on the concept of transferability. Transferability or external validity refers to the extent to which findings of a study applies to other areas of research [44]. In regards to transferability, the researchers developed the research questions by accessing extant literature. Likewise, the research focus served to add to the current literature and assist in addressing apparent gaps regarding obesity and MFT education. In addition, through the discussion of the results and future directions of research, the authors provided recommendation's that may be applicable to other groups (clinical social workers, professional counselors, and psychologists) regarding obesity education and clinical practice. Additionally, the results can provide preliminary data for larger studies (quantitative or mixed methodological studies) to further investigate the topic of obesity education within graduate schools in other fields such as medicine, nursing, and physical therapy, among others.

\section{Recruitment}

After gaining Institutional Review Board approval to conduct the study, the investigators (some of whom are current MFT students or MFT faculty) used their social and professional networks to advertise for the study. Members of the research team contacted their local MFT chapters affiliated with the national organization, the American association for marriage and family therapy. Authors also used snowball sampling [45] to identify recent graduates who might have an interest in the study. The research team sent fliers via email and text messages to individuals who might have potential interest in the study or knew of others who might be eligible for the study. Text messaging 
represented another effective form of recruitment used to reach participants in research [46].

The authors also recruited online using social media, mainly Facebook, as they posted the recruitment flier on their personal or professional Facebook pages. Likewise, authors sent the recruitment fliers via direct messaging on Facebook as well as on some public Facebook pages typically accessed by marriage and family therapists. Utilizing Facebook as well as other social media has worked well in previous research to solicit potential research subjects [47]. The recruitment flier included contact information of the PI to assist potential candidates for the research. Once individuals contacted the PI and expressed an interest in the study, the PI determined their eligibility and then scheduled a date and time for an interview.

\section{Data Collection}

Respondents who expressed an interest in the study reviewed the eligibility requirements and returned a signed informed consent document to the PI via email. To participate in the study, individuals had to self-report their status as recent graduates of a COAMFTEapproved marriage and family therapy program (within the last five years), in addition to meeting age requirements for the study (older than 18) and requirements regarding residency (U.S. based program). Interviews took place during times deemed convenient for participants and started with an overview of the study. During this interaction, the authors reviewed the purpose of the study and important information regarding informed consent and compensation. For the study, participants received a $\$ 20$ stipend. The authors then asked participants to select a mock name (pseudonym) to protect their confidentiality. After selecting a pseudonym, participants answered demographic questions focused on personal history and institution(s) attended, as well as individual and family history related to the topic of the study. After logging the participants' responses to the demographic questions, the interviewers verbalized that they would start audio recording and then proceeded to ask the formal interview questions designed to explore the meaning of obesity among marriage and family therapists. To review selected interview questions, see appendix A: Selected interview questions. For this study, authors based the sample size on data saturation as interpreted by two members of the research team. After each interview, two members of the research team reviewed the data and made notes before moving on to the next interview. Two researchers compared each interview to the previous interview, and interviews ceased after the two determined that additional interviews would yield redundant results [48].

\section{Analysis \\ The research team uploaded the audio-recorded data into a password protected media sharing computer program for the purpose of archiving, sorting, and coding the data. Members of the research team transcribed the text from the audio files before engaging in the analysis process. Data analysis consisted of a two-pronged approach which included: 1 . each member becoming independently immersed in the data and coding data, and 2. members meeting as a group to assess preliminary findings and determine final themes.}

Immersion of the data consisted of the PI and three of the coinvestigators independently reviewing the data. During the analysis stage, each individual listened to the interviews multiple times while taking notes regarding content. Consistent with phenomenology [49], research members focused on data that illuminated how Marriage and
Family Therapists construct meaning in regards to the concept of obesity. The researchers compiled notes regarding content, paying close attention to interview questions as well as key phrases and statements used by participants. In particular, each member coded the data using Microsoft Word to highlight codes and make notes in the margins.

According to Bradley et al. [50] coding provides the analyst with a formal system to organize the data, uncovering and documenting additional links within and between concepts and experiences described in the data. Likewise, codes represent "labels," which are assigned to whole documents or segments of documents (i.e., paragraphs, sentences, or words) to help catalogue key concepts while preserving the context in which these concepts occur (p.1761).

After members individually identified codes, they attempted to group the codes into categories if and when possible, and then used these categories to develop overarching themes. The reviewers utilized a constant comparative approach [37] to assess commonalities among the participants and to develop preliminary codes and to determine final emergent themes.

The second part of the analysis phase of the study consisted of a team meeting where the PI and the co-investigators specifically involved in the coding process met to discuss their initial thoughts, reactions, and perspectives regarding the data. Members presented their individual findings as well as reviewed how they conceptualized the emerging themes. After members presented their independent results, they engaged in a collaborative discussion regarding developing a master list of emergent themes. This discussion spanned three meetings among team members before solidifying the study's emergent themes. The research team also addressed areas needing clarification in follow-up meetings and discussed any negative or unique cases that surfaced.

\section{Demographics}

A total of 15 MFTs participated in the study, and researchers attempted to recruit individuals from various parts of the United States. The study consisted of a gender breakdown of five males and 10 females. Participants self-identified their race/ethnicity, with 11 identifying as African American, with the remaining four participants reporting "various races and ethnicities." The average age of participants was 32 . The average height of participants was 62.2 inches, ranging from 58 to 73 inches. With an average weight of 191.4 pounds, the participants' weight ranged from 125 pounds to 313 pounds. With an average body mass index of 30.767 (class I obese), participants ranged from 22.5 (normal weight) to 57.2 (class III obese). In the study, seven MFTs reported having a personal history of issues related to obesity, weight, body image, or food compulsion, with three specifically mentioning hypertension. Likewise, 11 MFTs reported that obesity, weight, body image, or food compulsion directly impacted their immediate or extended family members, with diabetes and hypertension being the most prevalent.

Theme 1: "Being unhealthy, being overweight", "obesity means to be significantly overweight. Obesity is mainly viewed within the context of the medical model, using body size 'normal standards' as the main way that obesity is viewed" (John).

"Being unhealthy, being overweight" represented the study's first emergent theme. Researchers asked participants in the study to define obesity and discuss how they understood it within the context of clinical work. When asked about the term "obesity," all of the 
participants $(\mathrm{n}=15)$ referred to obesity as a medical disease and used phrases such as "being unhealthy" and "being overweight". Some individuals also referred to obesity as "having an excess amount of body weight" or as being determined by one's Body Mass Index. When asked about how participants defined obesity, Wadell stated the following:

"Obesity is when a physician does an evaluation and tests your BMI. It may be anything over 32 . It means when normal things become exhausting (walking up stairs and getting dressed) and you become immobile. It also means having excess weight".

Likewise, brian, another participant, stated, "obesity is defined as being very unhealthy, inactive, and having poor eating habits over a long period of time". Furthermore, another participant, Cara, stated, "There is the medical definition of obesity; it is related to body weight and health, as well as other things." Grace, another participant, suggested that "Obesity is being overweight to the point where your health is affected and it is physically obvious." Whereas, vela, another participant, suggested that obesity is "Not being able to participate in everyday activities and not being able to have a good quality of life".

Researchers also asked respondents to describe how they understood obesity within a systemic framework. During the interview, a majority of the participants $(n=12)$ provided information about couples and families and reported that obesity can have a negative impact on the entire family system, specifically on romantic relationships. During Brian's interview as he discussed obesity, he reported: "I think it can have a huge impact on the family and relationships because depending on that person's health, you know, they can become ill or hospitalized, and it brings stress on the family financially. It can also result in death with that person not being here anymore or it can spiral other family members into a similar pattern".

Another interesting response during the interview came from TC, who stated the following: "Obesity can be, for some, a form of protection; so that they don't have to interact with others and sometimes it could be a reflection of a lack of discipline and not knowing how to take care of yourself"'.

Several participants $(n=10)$ discussed how obesity can impact a person's self-esteem. For example, in Destiny's response to the question, "What does it mean to be an obese individual?" she stated that "It means to feel some sort of shame and to have people look at you, feeling shame if you are a woman, in regards to no man will want you". Another participant, Waddell, communicated a similar perspective and responded, "an obese person is burdened". One participant discussed how obesity can impact intimate relationships. For example, Ryan stated the following:

"It can definitely cause issues, particularly if one is obese and the other person is an ideal body weight or doesn't have any struggles with body weight, guilt, or shame. A person may also feel as if they are not adequate for their spouse and not attractive to their partner".

Lionel also discussed the role that obesity has on families and stated the following: "Obesity can play a major role among family and romantic relationships (i.e., marriage), and it can affect both aspects similarly. In some instances, the partner has to help manage the circumstances of the other person who is obese or with health problems".

John also reported similar aspects and articulated that "Families deal with the stigma of obesity as well as physical health issues, and potentially obesity can impact sexual relationships".
Theme 2: Personal knowledge and self-perceptions

"My parents are overweight, and I see how that can affect day-today interactions with people and day-to-day how it can affect what you eat and what clothes you wear" (Cindy).

The research team determined "Personal Knowledge and SelfPerception," as the second theme that emerged. Participants $(n=12)$ reported that their knowledge and perspectives regarding obesity emerged from their own experiences of weight issues as well as problems related to obesity that family members or friends experienced. In particular, individuals reported that exposure to obesity in their personal lives, more so than their professional lives, have allowed them to empathize with clients who faced weight challenges. For example, when a researcher asked cindy to express her familiarity with working with obese clients, she mentioned how she personally exercises and tries to maintain a healthy weight. However, she added that her parents are overweight and then stated that she could understand how obesity may affect an obese person's daily interactions with people and personal preferences regarding food and clothing selections. Due to her personal experiences and knowledge in motivational interviewing, Cindy stated that she is very knowledgeable about obesity and expressed a sense of confidence in her interactions with obese clients and her ability to provide support for them. Another participant, Brian, mentioned his familiarity with obesity and selfconfidence in working with obese clients due to his personal experiences with family members who have had gastric bypass surgery for weight loss, while participant, Lionel, mentioned a heightened level of comfort in working with obese individuals due to his own personal struggles with his weight.

Although a few participants mentioned feeling comfortable working with obese populations, they also reported a lack of professional knowledge about obesity. During the study, researchers asked participants if they could describe specific weight-loss interventions (specific diets, nutrition plans, and food supplements), prescribed and over-the-counter medications (i.e., Orlistat, Dexatrim, Phentermine), weight-loss procedures (i.e., liposuction), surgery (i.e., lap band, gastric bypass), lifestyle modifications, and medications that may contribute to weight gain (i.e., corticosteroids, antipsychotics and antidepressants), general Body Mass Index guidelines (underweight, overweight, obese). An overwhelming majority $(n=12)$ failed to name more than one or two in each category. In the study, participants $(n=12)$ described more than two over-the-counter diet programs and at least one type of surgical intervention. However, when asked to describe the diet plan or provide details about surgical interventions, participants struggled. Respondents could not articulate general knowledge regarding the topic of obesity related to Body Mass Index, weight-loss interventions, medication, or other types of interventions.

Personal knowledge and self-perceptions of obesity also impacted how participants viewed themselves. During the interview, researchers asked participants if they knew their Body Mass Index. In the study, the majority of the participants $(n=14)$ reported that they did not know their body mass index. Likewise, only one individual (Destiny) reported that she was aware of her BMI and noted that she knew this since she recently had an appointment with her medical provider who discussed her BMI. In the study, members of the research team also asked participants how they self-identified in terms of their weight and body size. In particular, individuals responded to the following question: "Do you identify as being underweight, having a normal body weight, being overweight, or being obese?" In the study, only one individual self-identified as "obese" although based on BMI, five 
participants fell into the category of "obese." In the study, a total of eight participants self-identified as "overweight". However, four of the individuals identified as "overweight" which would technically classify them as "obese". Likewise, six participants in the study identified as having a "normal body weight"; however, four of the six fell into the category of "overweight," according to Body Mass Index.

In the study, several participants $(n=8)$ self-identified in a way that was incongruent with their Body Mass Index. When researchers asked participants about BMI, at least two individuals who reported identifying as overweight mentioned an awareness that, based on BMI, they would fall into the category of "obese". Two participants reported that due to the stigma of obesity, they preferred not to apply the term when self-identifying. For example, when asked to self-identify, John stated, "Ah (laugh), I identify actually as overweight, just because I think of the stigma attached to the word obese. However, medically, you know it would be obese, but I identify as overweight".

\section{Theme 3: Lack of knowledge and confidence}

"Um, I haven't had any obese clients, but I would say that I wouldn't come into the room thinking that I would need to treat them differently than I would treat any of my other clients" (Cindy).

The research team cited "Lack of knowledge and confidence" as the third theme that emerged in the study. During the interviews, some participants $(n=9)$ expressed that marriage and family therapists are familiar or somewhat familiar with the term "obesity" and some of the issues that obese patients may face in life; others expressed less familiarity. In the study, participants $(n=10)$ reported that they learned about issues that obese individuals may face from other colleagues, the Internet, family members, friends, and the media. During the participants' interviews, several of the individuals $(n=10)$ noted that they knew very little about obesity as it relates to therapy and reported having limited exposure to the topic in their master's training program.

During the interview process, one participant $(n=1)$ mentioned that while he did not have exposure to working with obese clients, a few of his classmates did internships in a pediatric obesity clinic or a primary healthcare facility and therefore had some exposure. For example, during interviews, Wadell stated, "I am pretty familiar with my current position. I work in an outpatient center. I work with people from a biospychosocial level. We have hypertension, high cholesterol, diabetes, and chronic heart failure. We see that stuff every day". Other participants reported having some exposure tangentially to working with clients who presented with comorbidities such as diabetes and hypertension. However, participants reported that they did not discuss obesity or comorbidities in-depth, outside of teaching coping skills. In addition, a few participants $(n=3)$ mentioned that they engaged in some discussion about the topic of obesity in their graduate programs. For example, during the interviews, Ryan reported that teachers did not cover obesity in his MFT training program, with the exception of minimal discussions in an addictions course and during clinical supervision. However, all participants $(n=15)$ reported that the instructors in their graduate level training programs did not specifically address the topic of obesity in a meaningful way.

During the study, researchers asked participants if they learned about various weight-loss interventions or if they had the opportunity to work with patients who reported with issues related to obesity and an overwhelming majority $(\mathrm{n}=10)$ reported zero chances to work with clients regarding issues of obesity. Likewise, participants reported that, for the most part, they did not gain experience applying traditional MFT theories to obesity and weight-related issues during didactic and clinical training in their graduate programs. Although participants could verbalize how they might apply traditional theories (modern and post-modern) with obese patients, they reported no formal training in discussing theory and obesity in their graduate programs. The majority of the participants $(n=12)$ noted that they would provide limited feedback to clients dealing with issues related to obesity due to their own lack of knowledge and formalized training. During the interviews, participants repeatedly discussed their lack of confidence in working with clients regarding weight-related concerns. For example, when asked about level of comfort in working with clients, Grace stated the following: I am not that confident with my current knowledge, but I would take the initiative to research some information. I would contact a doctor that I work for or research online. I would start with Googling obesity and the effects. I have not faced any challenges working with obese clients and therapy. I also have not worked with clients who want to specifically lose weight.

Furthermore, participants reported that they felt unprepared to address some of the issues that obese individuals might express in a therapy setting. For example, when asked about her level of comfort in interacting with obese clients, Bridget stated, "I am not 100 percent confident working with a client who is trying to lose weight, I would like more training in the area". Other participants communicated less confidence in working with obese individuals due to their inadequate knowledge of obesity and weight-loss guidelines, as well as interventions for weight loss. One participant, Varnes, stated that she has never interacted with any obese clients in her current practice in addition to a lack of awareness of interventions or guidelines related to obesity; as a result, she expressed an insecurity regarding her ability as a therapist to relate to this specific population.

Although participants $(\mathrm{n}=10)$ primarily mentioned not having formalized training in the area of obesity, many $(n=14)$ expressed a desire to gain more competence in the area. Likewise, most participants expressed that further education in obesity would increase their knowledge about obesity and confidence in working with obese clients. They also mentioned that collaborating with other healthcare professionals, such as physicians, would increase their expertise in the field as therapists. In addition, participants stated that increased knowledge regarding obesity terminology, receipt of direction towards obesity guidance and resources from a clinical and non-clinical perspective, and engagement in multidisciplinary collaborations with other healthcare professionals would, in turn, increase their comfort level and confidence when working with obese clients.

\section{Discussion}

The purpose of the study was to explore the meaning of obesity among marriage and family therapists. Therefore, the focus of the study was on asking questions that would illuminate attitudes, thoughts, perceptions, and experiences of marriage and family therapists. The themes that emerged in the study ("being unhealthy, being overweight," "personal knowledge and self-perceptions," and "lack of knowledge and confidence") directly related to the research questions. MFT participants' knowledge of the condition of obesity stems from vague limited general medical knowledge and, at times, personal experiences. While personal experiences can enhance knowledge, objective education may be needed to work with clients effectively and competently. The research for this study was consistent with other scholars who have explored the topic of obesity knowledge among medical students [51], medical residents, [52], nursing staff, 
exercise professionals, and behavioral health specialists [53], specifically related to a need for increased education.

The research team's findings highlight the fact that recent graduates' understanding of obesity rests mainly on the medical understanding of this condition which takes into account some understanding of body size and ideal weight. While a few recent graduates had a general understanding of the physical characteristics of obesity (i.e., BMI), their responses solidified the mindset that a more in-depth knowledge of obesity could help ensure that MFTs understand obesity as well as their potential role in addressing this issue. In addition, this study warrants that the scope of practice implemented by MFTs regarding obesity deserves more attention.

Similar to previous studies [7], some participants in the study struggled with the notion of what their roles entail in working with obese clients. This study also highlights and confirms other findings that reflect that the role of bias merits consideration when developing training programs, some of which may be shaped by personal experiences. These findings remain consistent with previous research that indicated that a lack of clinical training and bias towards persons experiencing weight-related issues, such as obesity [7], can serve to influence how therapists interact with obese patients in their future work. While revamping the training process [6] prefaces the idea that instructors in the MFT programs must ensure that student clinicians receive the necessary tools to provide sensitive treatment to clients impacted by obesity, it is also pertinent that future research focus not only on biases about clients, but therapists' own personal perceptions and experiences regarding weight as well. The complex issue of obesity requires that clinicians move beyond merely the physical presence that may dominate the other effects of the condition. Furthermore, clinicians must consider emotional, psychological, and familial implications of obesity, while balancing their own personal beliefs and perceptions. Because of these complexities, recent graduates require better preparation in their training programs. Likewise, participants voiced a concern for additional training to better prepare clinicians to meet the needs of this growing population.

\section{Recommendations for Training}

Based on the results of the study, MFTs and presumably other mental health professionals would benefit from enhanced training regarding obesity and weight loss within the context of individual, couple, and family relationships. Categorizing obesity as one of the most challenging issues to combat due to its impacts in the areas of physical health and mental health in America and abroad, the authors recommend training via a course in graduate school as part of a standard MFT curriculum, or as an elective course that students can take outside of the standard MFT curriculum, perhaps through a public health, health psychology, nursing, pharmacy, or medical school training program. The authors also suggest that this training addresses both didactic and clinical/experiential perspectives and recommend that it covers biological, psychological, and relational aspects of obesity.

Those who have completed their degrees could participate in a postgraduate training curriculum offered in a variety of formats and developed to address the needs of therapists who have already finished their education. The authors also argue that a course within graduate school should also include or at least consider personal experiences of obesity and how this may impact epistemology, attitudes, and beliefs about treatment. In addition to didactic training, a course that specifically highlights potential issues in supervision may also be worth considering when conceptualizing obesity education, as recent graduates may have individual or family experiences with obesity and comorbidities (diabetes mellitus, hypertension, and hypercholesterolemia) and weight-related mental health concerns (depression, bulimia, binge- eating, negative body image) that may impact their education and ultimately their work with clients.

\section{Limitations}

A number of limitations surfaced for the research team during the course of this study. As a qualitative study, this research is subject to the limitations related to a lack of generalizability. However, the authors contend that the results can readily apply when considering some dimensions of MFT education. The authors noted that one limitation of the study emerged from the fact that the authors focused on master's level training, without addressing training at the doctoral level. Including doctoral level therapists in the research data may provide an additional perspective about the needs of therapists in terms of clinical training. However, the Master's degree in MFT constitutes a terminal degree and carries with it a license to practice. While some MFT doctoral training programs provide enhanced training in clinical intervention, such as a doctorate in MFT (DMFT in most states or a Ph.D in MFT in California), many doctoral programs (Ph.D.) focus on research rather than clinical training. Therefore, including a focus on obesity for the terminal degree may make more sense when considering direct clinical practice.

Another limitation of the study cited by the researchers included the sample, which consisted of a majority of African American therapists $(n=11)$. Despite this research study's limitations, the authors believe the findings add to the literature regarding obesity and the field of MFT training, which can be useful for program directors and other faculty as they consider ways to expand the knowledge base of therapists among COAMFTE-accredited master's programs. Furthermore, one limitation of the study was the small sample size. However, the authors contend that the research can be used as a preliminary study for a larger study. Likewise, the authors argue that sample size in qualitative research is based on saturation [54] and the purpose of the study was to explore a phenomenon, not produce results that are generalizable.

\section{Future Research}

Although this project utilized a qualitative approach to gain an understanding of the opinions, perspectives, and beliefs among marriage and family therapists, this study provides insights into developing ideas and hypotheses for potential quantitative research that further explores the knowledge of obesity and other health-related medical concerns. Furthermore, numerous perceptions about body mass index from participants suggest the need to consider personal experiences of obesity and perceptions of BMI.

The research team contends that further studies exploring interdisciplinary collaborations between marriage and family therapy, pharmacy, and medical educators may prove to be beneficial when exploring obesity competency in education. This study provides a good starting point for assessing the need of interdisciplinary collaborations that will lead to the development of curricular changes and new strategies for teaching core and elective competencies in marriage and family therapy programs, in addition to improved patient health outcomes. Interdisciplinary collaborations may also lead to advanced expertise and knowledge that may have been previously missed in 
didactic or experiential settings conducted solely by marriage and family therapists. These collaborations may also lead to further research that assesses the awareness and a better understanding of obesity among other mental health professionals (clinical social workers, psychologists) and allied health professionals (pharmacists, nurses, medical doctors, dietitians). Additional research that may be warranted include a large quantitative study that specifically assesses the general knowledge of obesity and weight-related behaviors of MFTs that may cover such topics as Body Mass Index, medical treatment, nutrition, eating disorders, surgical procedures, medication, and behavioral interventions. A formalized assessment or established and validated instrument could provide an objective baseline measure that could be used when determining the exact need of MFT graduates. Future research could also include assessing the personal experiences of therapists regarding obesity and eating disorders, in addition to obtaining collateral data from clients and medical professionals about their thoughts regarding knowledge that MFTs may need to possess when working with clients who present with concerns regarding weight-related behaviors.

The researchers have produced results that impact not only the field of marriage and family therapy, but also in collaborative health in general as it adds the voices of marriage and family therapy graduates to the discussion in a way that has not been done before. Although previous researchers have explored the topic of obesity among marriage and family therapists [6], to date, there have not been a significant number of researchers who have utilized qualitative methodological approaches such as semi-structured interviews and focused on clinician's knowledge of obesity.

\section{References}

1. Dietz WH (2015) The response of the US centers for disease control and prevention to the obesity epidemic. Annu Rev Public Health 36: 575-596.

2. Hruby A, Manson JE, Qi L, Malik VS, Rimm EB, et al. (2016) Determinants and consequences of obesity. Am J Public Health 106: 1656-1662.

3. Lee G (2015). Obesity, the epidemic that CAN be stopped? Journal of Advanced Nursing 71: 1971-1972.

4. Ogden CL, Carroll MD, Fryar CD, Flegal KM (2015) Prevalence of obesity among adults and youth: United States, 2011-2014.

5. Fierabracci P, Tamberi A, Santini F (2015) Obesity-related comorbidities. In Minimally invasive bariatric and metabolic surgery.

6. Pratt KJ, Holowacz E, Walton NL (2014) Marriage and family therapist's perspectives on treating overweight clients and their weight-related behaviors. Ame J Family Ther 42: 364-385.

7. Cravens JD, Pratt KJ, Palmer E, Aamar R (2016) Marriage and family therapy students views on including weight bias training into their clinical programs. Contemporary Family Therapy 38: 210-222.

8. Pratt KJ, Palmer E, Cravens JD, Ferriby M, Balk E, et al. (2016) Marriage and family therapy trainees reports of explicit weight bias. J Marital Fam Ther 42: 288-298.

9. Simon GE, Von KM, Saunders K, Miglioretti DL, Crane PK, et al. (2006). Association between obesity and psychiatric disorders in the US adult population. Arch Gen Psychiatry 63: 824-830.

10. Dankel SJ, Loenneke JP, Loprinzi PD (2016) Mild depressive symptoms among Americans in relation to physical activity, current overweight/ obesity, and self-reported history of overweight/obesity. Int J of Behav Med 23: 553-560.

11. Palmeira L, Pinto-Gouveia J, Cunha M (2016) The role of weight selfstigma on the quality of life of women with overweight and obesity: A multi-group comparison between binge eaters and non-binge eaters. Appetite 105: 782-789.
12. Gibson LY, Byrne SM, Davis EA, Blair E, Jacoby P, et al. (2007) The role of family and maternal factors in childhood obesity. Med J Aust 186: 591-595.

13. Lim CS, Gowey MA, Janicke DM (2014). Behavioral family treatment of pediatric obesity in an underserved community-based setting: A case study demonstrating barriers to treatment effectiveness. Clin Practice Pediatr Psychol 2: 236-249.

14. Edmunds L, Waters E, Elliott EJ (2001) Evidence based paediatrics: Evidence based management of childhood obesity. BMJ 323: 916-919.

15. Kitzmann KM, Beech BM (2006) Family-based interventions for pediatric obesity: Methodological and conceptual challenges from family psychology. J Fam Psychol 20: 175-189.

16. Nowicka P, Flodmark CE (2011) Family therapy as a model for treating childhood obesity: Useful tools for clinicians. Clin Child Psychol Psychiatry 16: 129-145.

17. Nowicka P, Pietrobelli A, Flodmark CE (2007). Low-intensity family therapy intervention is useful in a clinical setting to treat obese and extremely obese children. Int J Pediatr Obes 2: 211-217.

18. Engebretsen SR, Early KB, Sorrells R, Joyce P, Sanchez M, Gottlieb K, et al. (2016). Longitudinal quality of life improvement after family based intervention in youth with obesity. FASEB 36: 422-424.

19. Sacher PM, Kolotourou M, Chadwick PM, Cole TJ, Lawson MS, et al. (2010). Randomized controlled trial of the MEND program: A familybased community intervention for childhood obesity. Obesity 18: S1.

20. Moore D, Cooper C, Williams T, Zwiestera K, (2017) Life after NBC's "the biggest loser": The experiences and perspectives of former reality TV contestants. The Qualitative Report, 22: 683-711.

21. Pratt KJ, Lamson AL, Collier DN, Crawford YS, Harris N Gross, et al. (2009). Camp golden treasures: A multidisciplinary weight-loss and a healthy lifestyle camp for adolescent girls. Families, Systems, and Health, 27: 116 .

22. Aramburu AC, Larsen B (2016). Contextual care of the patient following weight loss surgery: Relational views and maintenance activities of couples. J Am Assoc of Nurse Pract 29: 17-25.

23. Ferriby M, Pratt KJ, Balk E, Feister K, Noria S, et al. (2015) Marriage and weight loss surgery: A narrative review of patient and spousal outcomes. Obes Surg 25: 2436-2442.

24. Moore D, Cooper C (2016) Life after bariatric surgery: Perceptions of male patients and their intimate relationships. J Marital Family Ther 43: 495-508.

25. Moore D, Few-Demo A (2017) The meaning of bariatric surgery for male patients: Self-concept and the search for masculinity. Journal of Men's Health, 13: e25-e36.

26. Pories ML, Hodgson J, Rose MA, Pender J, Sira N, et al. (2016) Following bariatric surgery: An exploration of the couples' experience. Obes Surg 26: 54-60.

27. Brooks J, Moore D (2016) The impact of childhood experiences on perceptions of health and wellness in African American young adults. Journal of African American Studies, 20: 183-201.

28. Doherty WJ, Jill E, Harkaway (1990) Obesity and family systems: A family FIRO approaches to assessment and treatment planning. J Marital Family Ther 16: 287-98.

29. Harkaway JE (1986) Structural assessment of families with obese adolescent girls. J Marital Family Ther 12: 199-201.

30. Harkaway JE (1987) Family intervention in the treatment of childhood and adolescent obesity. In JE Harkaway (Ed), Eating disorders.

31. Harkaway JE (2000) Obesity and systems research: The complexity of studying complexities. Families Systems Health 18: 55.

32. Mcvoy JH (1987) Family fat: Assessing and treating obesity within a family context. 38. In JE Harkaway (Ed), Eating disorders.

33. Tyndall LE, Hodgson JL, Lamson AL, White M, Knight SM (2012) Medical family therapy: Charting a course in competencies. Contemporary Family Therapy 34: 171-186. 
Citation: Moore D, Williams L, Kimbrough A, Taylor C, Jacob M, et al. (2017) The Meaning of Obesity among Marriage and Family Therapists. J Nutr Weight Loss 2: 108.

Page 9 of 9

34. Hodgson J, Lamson A, Mendenhall T, Crane R (2012) Medical family therapy: Opportunity for workforce development in healthcare. Contemporary Family Therapy 34: 143-146.

35. Dahl CM, Boss P (2005) The use of phenomenology for family therapy research. Research Methods in Family Therapy 2: 63-84.

36. Barriball KL, While A (1994) Collecting data using a semi-structured interview: A discussion paper. J Adv Nursing 19: 328-335.

37. Leech BL (2002) Asking questions: Techniques for semi structured interviews. Political Science and Politics, 35: 665-668.

38. Hanna P (2012) Using internet technologies (such as skype) as a research medium: A research note. Qualitative Research, 12: 239-242.

39. Carey MA (1994) The group effect in focus groups: Planning, implementing, and interpreting focus group research. In J Morse (Ed), Critical issues in qualitative research methods.

40. Marshall MN (1996) Sampling for qualitative research. Fam Pract 13: 522-525.

41. Golafshani $N$ (2003) Understanding reliability and validity in qualitative research. The Qualitative Report 8: 597-606.

42. Mauthner NS, Doucet A (2003) Reflexive accounts and accounts of reflexivity in qualitative data analysis. Sociology 37: 413-431.

43. Wimpenny P, Gass J (2000) Interviewing in phenomenology and grounded theory: Is there a difference? Journal of Advanced Nursing 31: 1485-1492.

44. Shenton AK (2004) Strategies for ensuring trustworthiness in qualitative research projects. Education for Information 22: 63-75.

45. Coyne IT (1997) Sampling in qualitative research. Purposeful and theoretical sampling; merging or clear boundaries? J Adv Nursing 26: 623-630.
46. Leong KC, Chen WS, Leong KW, Mastura I, Mimi O, et al. (2006) The use of text messaging to improve attendance in primary care: A randomized controlled trial. Family Practice 23: 699-705.

47. Bhutta CB (2012) Not by the book: Facebook as a sampling frame. Sociological Methods \& Research 41: 57-88.

48. Walker JL (2012) The use of saturation in qualitative research. Can J Cardiovasc Nurs 22: 37-46.

49. Donalek JG (2004) Phenomenology as a qualitative research method. Urol Nurs 24: 516-517.

50. Bradley EH, Curry LA, Devers KJ (2007) Qualitative data analysis for health services research: Developing taxonomy, themes, and theory. Health Services Research, 42: 1758-1772.

51. Swift JA, Sheard C, Rutherford M (2007) Trainee health care professional knowledge of the health risks associated with obesity. J Human Nut Diet 20: 599-604.

52. Block JP, Desalvo KB, Fisher WP (2003) Are physicians equipped to address the obesity epidemic? Knowledge and attitudes of internal medicine residents. Preventative Medicine 36: 669-675.

53. Bleich SN, Bandara S, Bennett WL, Cooper LA, Gudzune KA (2015) U.S. health professional views on obesity care, training, and self-efficacy. American Journal of Preventive Medicine, 48: 411-418.

54. Guest G, Bunce A, Johnson L (2006) How many interviews are enough? An experiment with data saturation and variability. Field Methods 18: 59-82. 